\title{
Effectiveness and Acceptance of Adaptive Intelligent Speed Adaptation Systems
}

\author{
Stephen Arhin, Azim Eskandarian, Jeremy Blum, Pierre Delaigue, \\ and Damoon Soudbakhsh
}

\begin{abstract}
Intelligent speed adaptation (ISA) systems face significant consumer acceptance hurdles that limit the likelihood of widespread adoption, particularly in the United States. However, if these systems are designed as speed management systems rather than speed limiting systems, with adaptability to individual driving behavior, they may be more likely to meet with consumer acceptance. The results of a fixed-based driving simulator experiment that tested the acceptance and effectiveness of a new type of ISA, called an Advanced Vehicular Speed Adaptation System (AVSAS), are reported. The results of the experiment showed that AVSAS resulted in reductions in driver speeds across a range of roadway types. AVSAS is a speed management system that adapts to an individual driver's speed behavior and the current driving situation. AVSAS resulted in an average reduction of $5 \%$ of the maximum speeds and $3 \%$ of the average speeds of the drivers on four road segments. As expected, AVSAS did not reduce driver speeds as much as the mandatory control ISA system, and the experiment confirmed the results of tests conducted on ISA systems largely in Europe. Conversely, the results revealed that more participants were willing to purchase AVSAS compared with the information or mandatory ISAs. Although these results show the promise of a trade-off between system effectiveness and acceptability that has been missing in mandatory and information ISA research, AVSAS suggests that a range of ISA system design requirements could encourage the adoption of ISA systems in the United States.
\end{abstract}

Excessive speeding is responsible for an alarming number of fatalities and serious injuries. Research conducted by the National Highway Transportation Safety Administration (NHTSA) shows that approximately one third of annual fatal crashes involved at least one driver driving too fast for prevailing weather conditions or exceeding the legal speed limit (1). In 2003, crash data analyzed by NHTSA showed that approximately $15 \%$ of all crashes are speed related (1). NHTSA also estimated that speed-related crashes cost society more than $\$ 30$ billion a year. On average, approximately 1,000 fatalities are reported every month in speed-related crashes.

Traditional approaches to reducing driver speeds have limited effectiveness both in terms of duration and location. For example, studies have shown that police speed enforcement efforts tend to reduce speeds only near the enforcement area and only for a limited

S. Arhin, A. Eskandarian, P. Delaigue, and D. Soudbakhsh, Center for Intelligent Systems Research, George Washington University, 20101 Academic Way, Ashburn, VA 20147. J. Blum, Department of Computer Science, Pennsylvania State University at Harrisburg, 777 Harrisburg Pike, Middletown, PA 17057. Corresponding author: S. Arhin, saarhin@gwu.edu.

Transportation Research Record: Journal of the Transportation Research Board, No. 2086, Transportation Research Board of the National Academies, Washington, D.C., 2008, pp. 133-139.

DOI: $10.3141 / 2086-16$ amount of time after a long-term enforcement effort (2). Similarly, roadway features designed to reduce speeding behavior tend to reduce speeds in a small area (3).

Intelligent speed adaptation (ISA) systems hold the promise of widespread and long-term improvement in speed behavior. These in-vehicle systems come in a range of configurations, from control systems that physically prevent the driver from exceeding a certain speed, to information systems, which simply warn a speeding driver.

To realize this promise, however, ISA systems must overcome significant hurdles in terms of consumer acceptance. Control systems, while effective in ensuring compliance with speed limits, are viewed extremely negatively by drivers. These systems are useful in some situations, including in fleet vehicles or for court-ordered driving remediation. However, it is unlikely that these systems would be voluntarily adopted for widespread use in private vehicles. Conversely, information systems are significantly less effective in reducing driver speeds. Moreover, although they are more acceptable to consumers, in most tests of information ISA systems in Europe, drivers have demonstrated resistance to the adoption of these systems.

This paper describes an Advanced Vehicular Speed Adaptation System (AVSAS), a new approach to ISA systems. This system, rather than attempting to simply limit the speed driven, offers a speed management system that adapts to drivers' speed behavior and the current roadway situation. AVSAS was evaluated in a fixed-based driver simulator experiment in which subjects drove without ISA, with a control or mandatory ISA system, with an information ISA, and with AVSAS. After the experiment, the subjects responded to a questionnaire that measured their acceptance of the different ISA systems. In addition, their driving behavior with each of the ISAs was analyzed.

\section{RELATED RESEARCH IN INTELLIGENT SPEED ADAPTATION}

Previous ISA systems can vary in three dimensions: the level of system persistence, the system speed limit, and the user interface (4):

- Level of system persistence. At one end of the spectrum, information systems can provide speed limit information and warnings when the speed limit is exceeded. At the other end, voluntary and mandatory ISA systems can have regulators, for example, a dead throttle mechanism that physically prevents the vehicle from exceeding a certain speed. Unlike mandatory systems, voluntary systems can be switched off or overridden by the driver.

- System speed limits. The simplest type is a fixed speed limit system, which has a single speed at which the ISA system will be activated. Variable speed limit systems use roadside beacons or 
global positioning systems and digital maps to activate the system on the basis of the speed limit for the current roadway. Dynamic speed limit systems allow for further refinement, varying the speed limit by roadway, weather conditions, or congestion.

- User interface. ISA systems provide visual displays, including head-up displays, dash-mounted light-emitting diode displays, enhanced speedometers; haptic displays, including stiffening of the accelerator pedal and virtual rumble strips; and auditory displays.

Previous studies of ISA systems have established a trade-off between system effectiveness and consumer acceptance (5). Mandatory control systems are more effective than voluntary control and information systems; mandatory systems are viewed unfavorably by drivers. Although voluntary and information systems are viewed more favorably than mandatory systems, these systems also face significant issues with consumer acceptance.

Numerous studies have shown that mandatory control intervention systems are much more effective in reducing speeding than voluntary control systems and information systems (6-9). Mandatory systems, with variable speed limits, can enforce complete compliance with speed limits. Analyses of voluntary systems, conversely, have shown that the percentage of time that these systems are actually turned on is very low. For example, in a large field operational test in Sweden, voluntary use of the ISA system was only $11 \%$ (10). Moreover, drivers are inclined to switch off the system as soon as they have the opportunity to speed (11).

Although effective in reducing speeding, mandatory systems face the largest hurdles in terms of consumer acceptance, with drivers who would benefit most from the ISA system most negatively inclined toward the system. Drivers have reported a range of negative reactions to mandatory, control ISA systems. Common reactions to these systems include increased stress, frustration, vulnerability, and low system satisfaction (12-14). These reactions have produced predictable consumer resistance to these systems. In one typical set of experiments, $38 \%$ to $70 \%$ of subjects would not put a speed limiter in their cars regardless of the cost (8).
Conversely, there is also evidence that there may be significant consumer resistance to voluntary and information systems. In one field operational test, $60 \%$ of drivers would not purchase an advisory system for $£ 50$ (8). In another advisory system with a voice warning, $39 \%$ of drivers found the system annoying (13).

\section{OVERVIEW OF AVSAS}

\section{Underlying AVSAS Architecture}

Generally, research has shown that the mandatory ISA system has been the most effective in reducing speeding, thereby increasing safety. However, these systems are the least accepted, especially by drivers who could benefit the most. Conversely, although information or warning ISA systems are slightly more acceptable to consumers, studies have shown that they are significantly less effective in reducing vehicle speeds than mandatory ISA systems. The new ISA, AVSAS, was developed with the aim of having both an effective and acceptable speed management and control system, which would primarily be based on drivers' speeding behavior under normal conditions.

On the basis of subjects' individual driving preferences determined during a first phase of simulator experiment, AVSAS defines a target speed (TS) for each driver. This threshold value corresponds to the average speed above the speed limit subjects usually prefer to drive. The TS is simply given by

$\mathrm{TS}=$ speed limit $+\Delta S$

where $\Delta S$ is the excess speed above the posted speed limit that a particular driver is comfortable driving.

When the TS is exceeded, AVSAS adapts its warning on the basis of individual speed profiles, driving scenario, and roadway geometry and condition. The overall architecture of AVSAS is shown in Figure 1. When drivers approach potential hazards that require

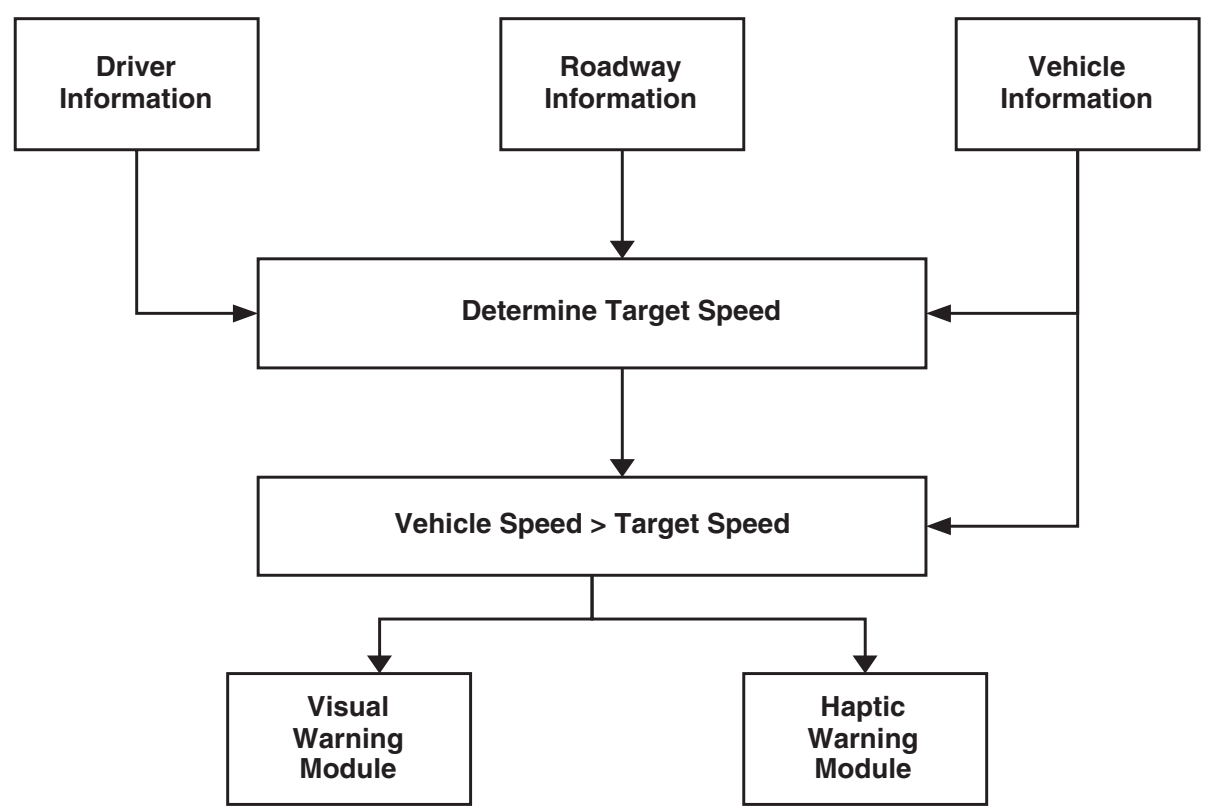

FIGURE 1 AVSAS architecture. 
TABLE 1 Warning Strategies in AVSAS During Free-Flow Driving

\begin{tabular}{lcll}
\hline Driving Scenario & Visual Mode & Haptic Stimuli & Information Icon \\
\hline Below speed limit & 0 & No & No \\
Above speed limit & 1 & No & No \\
Above target speed & 2 & Yes & Yes \\
Override mode & 2 & No & No \\
\hline
\end{tabular}

reduction in speed such as intersections, work zones, and curves, AVSAS response is individually customized and accounts for a number of factors including preferred deceleration rate and vehicle state at a given time.

\section{AVSAS Decision Algorithms}

The AVSAS learning and decision algorithm for system warnings was developed based on both individual current system state-ofthe-vehicle and driving styles. The algorithm determines the timing and modality of the warning to be provided. The driver has the option to turn the system off at any time and can override the system. Absent any approaching hazards (i.e., free-flow driving conditions), the warning strategy used is as presented in Table 1. Figure 2 shows the three different visual modes developed for this experiment, which are displayed within the speedometer area of the instrument panel, and provides a color-coded warning system that alerts the driver's speeding status.

A haptic warning is given to the driver when his or her current speed exceeds the target speed. This warning is achieved by changing the amount of force required to push the gas pedal or to maintain its given position when the target speed is exceeded. An active accelerator pedal allows a counterforce to be generated when the TS is exceeded. The amount of the counterforce generated is controlled by a software program, which allows the active accelerator pedal to be either configured as a control system with sufficient force to prevent the driver from speeding, or to be used as a warning system with lesser force that the driver can overcome. Feedback from the system, including resistance, vibration, and blocking, is generated on the basis of the changing resistance in the gas pedal; this is shown in Figure 3.

A host computer uses the AVSAS decision software module to control the amount of resistance in the gas pedal through an ana$\log$ computer servomechanism. The software also generates control signals for the servo amplifier, which in turn controls the amount of current in the servomotor. In the event of an approaching hazard that requires the driver to decelerate, a warning strategy, as listed in Table 2, is used.

The system was also designed to allow drivers to override the haptic warning by pushing the gas pedal against the feedback force. Once the TS is exceeded, the system can be put into an override mode by pushing the pedal all the way down. The system is reset when the speed drops below TS or there is a new approaching hazard.

\section{DESCRIPTION OF EXPERIMENT}

The experiment was conducted in two phases. The first phase involved driving under normal conditions without the assistance of any ISA system. This procedure established the baseline conditions for all the drivers. The drivers also completed surveys aimed at evaluating the fixed-based simulator for validation purposes.

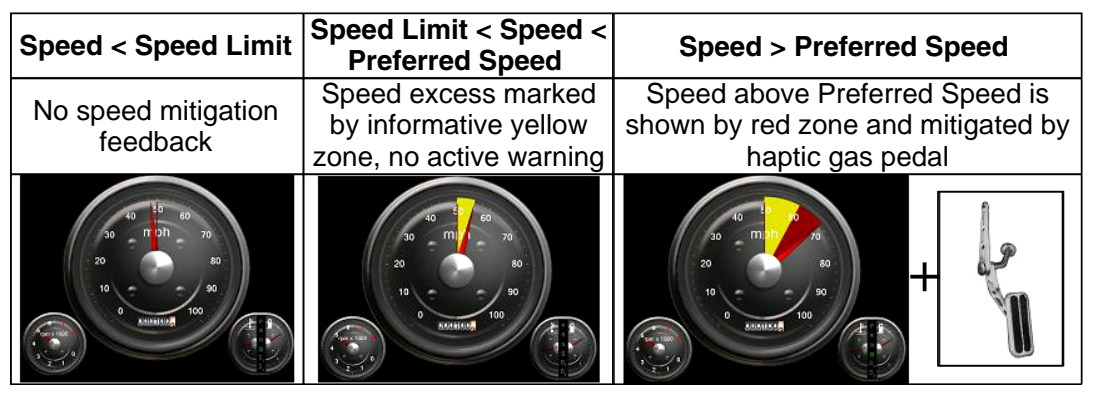

FIGURE 2 Visual warning modes.

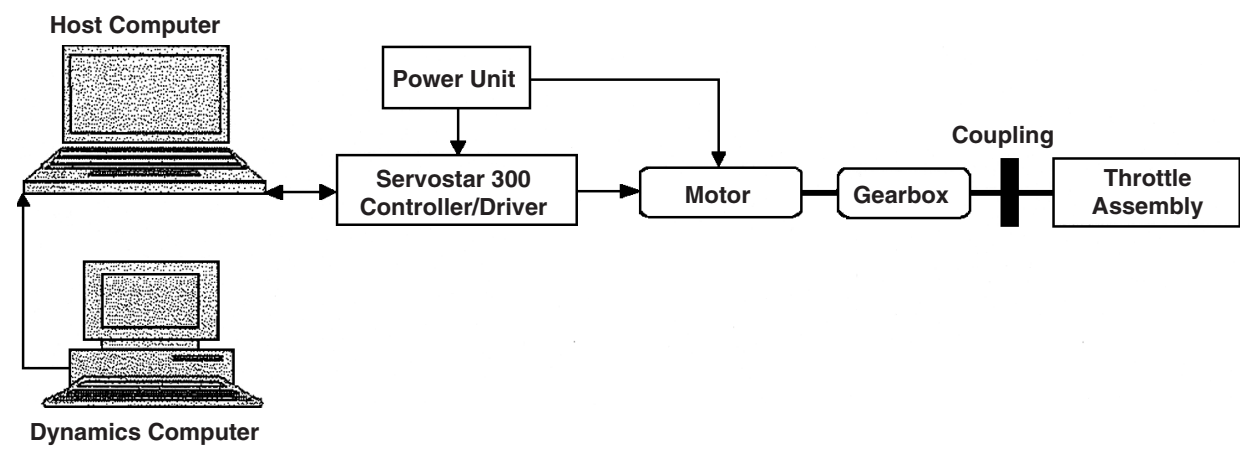

FIGURE 3 Schematic of active gas pedal system. 
TABLE 2 Warning Strategy Based on Vehicle Speed

\begin{tabular}{lcll}
\hline Driving Scenario & Visual Mode & Haptic Stimuli & Information Icon \\
\hline Below speed limit & 0 & Yes & Yes \\
Above speed limit & 1 & Yes & Yes \\
Above target speed & 2 & Yes & Yes \\
Override mode & 2 & No & Yes \\
\hline
\end{tabular}

In all, 32 regular licensed drivers aged 22 and 38 were recruited through advertisements to participate in the experiment voluntarily. Twenty-one of the 32 drivers completed the second phase of experiment, in which the three ISA systems were introduced in the order of information, mandatory, and AVSAS. The remaining subjects were unable to complete the experiment because they were not available. The parameterization of the AVSAS system for each driver was based on the baseline driving data recorded during the first phase of the experiment. The experiment was conducted between August 2006 and March 2007. The participants were given detailed instructions and an overview of each phase of the experiment.

For each driver, the speed of the vehicle throughout the experiment was recorded and retrieved for each phase. In addition, other corresponding variables, including the acceleration, posted speed limit, road type, and elapsed time were recorded and retrieved.

The variables analyzed were the maximum and average speeds of the drivers for six road classifications. The road classes, with their posted speed limits, are: freeways $(65 \mathrm{mph})$, collector roads $(45 \mathrm{mph})$, local roads (35 mph), residential roads ( $25 \mathrm{mph}$ ), rural roads (55 mph), and urban roads (35 $\mathrm{mph}$ ).

\section{EXPERIMENT RESULTS}

The simulator experiment confirmed that the new speed management approach in AVSAS represents a significant improvement in consumer acceptance, while still reducing driver speeds. The
TABLE 3 Comparing Individual Maximum Speeds (percentage of drivers)

\begin{tabular}{lcc}
\hline Segment & AVSAS $<$ NOISA & AVSAS $\geq$ NOISA \\
\hline Freeways (65 mph) & 80.95 & 19.05 \\
Collector (45 mph) & 66.67 & 33.33 \\
Local (35 mph) & 57.14 & 42.86 \\
Residential (25 mph) & 61.90 & 38.10 \\
Rural (55 mph) & 38.10 & 61.90 \\
Urban (35 mph) & 38.10 & 61.90 \\
\hline
\end{tabular}

analysis showed that AVSAS reduced maximum and average speeds on four of the six road classes. On average, maximum speeds were reduced by approximately $5 \%$ on four of the six road segments and average speeds were reduced by approximately $3 \%$ on the same segments.

\section{AVSAS Effectiveness}

The individual maximum and average speeds computed for each road classification and for each driver with the AVSAS technology were compared with the baseline driving scenario, which is referred to here as NOISA. The aggregate maximum and average speeds for all the drivers were also compared each type of roadway.

Table 3 shows a comparison of the percentage of drivers whose maximum speeds in AVSAS were less than those of the NOISA condition. These results, presented graphically in Figure 4, show that, of the six road classifications, maximum speeds were reduced on four segments. On average, for the four segments, maximum speeds in AVSAS were reduced by approximately $67 \%$ compared with the NOISA condition. With AVSAS, maximum speeds were reduced the most on freeways $(80.95 \%)$.

On average, the maximum speeds in AVSAS were reduced on four road segments compared with the NOISA condition, as shown in Table 4 . The reductions were determined to be statistically significant

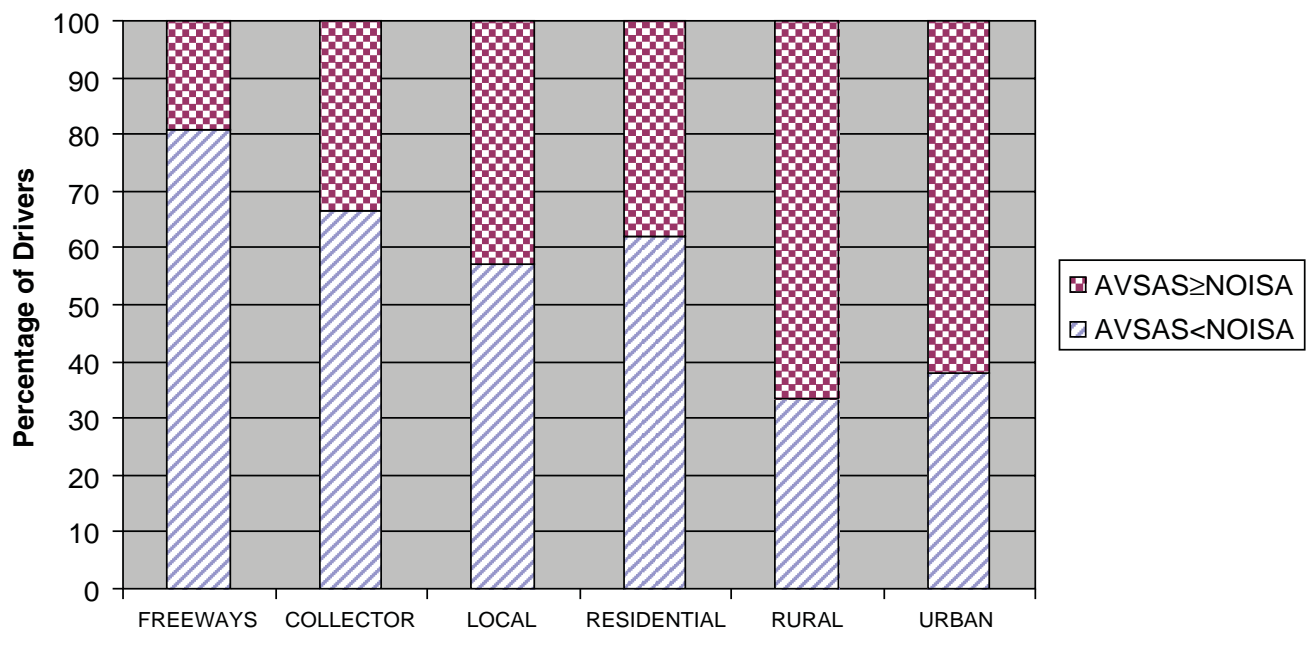

FIGURE 4 Comparing maximum speeds in AVSAS with NOISA. 
TABLE 4 Average Maximum Speeds (mph)

\begin{tabular}{lcc}
\hline Segment & AVSAS & NOISA \\
\hline Freeways (65 mph) & 72.75 & 78.98 \\
Collector (45 mph) & 54.32 & 56.01 \\
Local (35 mph) & 50.95 & 52.97 \\
Residential (25 mph) & 35.32 & 37.05 \\
Rural (55 mph) & 63.36 & 63.17 \\
Urban (35 mph) & 58.43 & 57.73 \\
\hline
\end{tabular}

TABLE 5 Comparing Individual Average Speeds [percentage of drivers]

\begin{tabular}{lcc}
\hline Segment & AVSAS $<$ NOISA & AVSAS $\geq$ NOISA \\
\hline Freeways (65 mph) & 47.62 & 52.38 \\
Collector (45 mph) & 61.90 & 38.10 \\
Local (35 mph) & 52.38 & 47.62 \\
Residential (25 mph) & 57.14 & 42.86 \\
Rural (55 mph) & 28.57 & 71.43 \\
Urban (35 mph) & 42.86 & 57.14 \\
\hline
\end{tabular}

for one of the four road types at a 5\% level of significance (freeways: $t=-2.63, p<.01)$.

Of the 21 drivers who completed both phases of the experiment, approximately $48 \%$ recorded reduced average speeds on all the six road types. These results are broken down by road type in Table 5 and Figure 5. The average speeds of all the drivers, as shown in Table 6, were reduced on four of the six road segments. These reductions, on the whole, represented approximately $3 \%$ over the NOISA condition. However, at 5\% level of significance, these reductions were not statistically significant.
TABLE 6 Average Speeds (mph)

\begin{tabular}{lcc}
\hline Segment & AVSAS & NOISA \\
\hline Freeways (65 mph) & 60.56 & 61.79 \\
Collector (45 mph) & 43.05 & 44.47 \\
Local (35 mph) & 38.46 & 39.22 \\
Residential (25 mph) & 23.73 & 24.57 \\
Rural (55 mph) & 54.73 & 52.23 \\
Urban (35 mph) & 37.37 & 37.35 \\
\hline
\end{tabular}

\section{Effectiveness of AVSAS Relative to Other ISA Systems}

The individual maximum and average speeds for AVSAS were also compared with those of the warning and mandatory ISA systems. As expected, and shown in Table 7, most of the drivers' individual maximum speeds in AVSAS were not reduced significantly when compared with the mandatory and warning ISA systems. As shown in Table 8, individual average speeds in AVSAS were not significantly reduced when compared with the mandatory and warning ISA systems, except on the freeway segment.

On average, the maximum and mean speeds with the mandatory and warning systems were generally found to be lower than those with the AVSAS system. A majority of the differences, particularly compared with the mandatory system, were found to be statistically significant at $5 \%$ level of significance.

The AVSAS technology was designed to provide drivers with the potential of exceeding the posted or prevailing speed limit up to a threshold. Thus, as expected, the drivers exceeded the speed limits more often than with particularly the mandatory ISA system, as well as the warning ISA system in some instances. In addition, the AVSAS technology provided the drivers with information on tolerable speeds above the posted speed limits, thereby allowing them to drive at those speeds rather than at the prevailing speed limits. As a result, the maximum and average speeds



FIGURE 5 Comparing average speeds in AVSAS with NOISA. 
TABLE 7 Comparing Individual Maximum Speeds (percentage of drivers)

\begin{tabular}{lcc}
\hline Segment & AVSAS $<$ Warning & AVSAS $<$ Mandatory \\
\hline Freeways (65 mph) & 33.33 & 14.29 \\
Collector (45 mph) & 23.81 & 0.00 \\
Local (35 mph) & 33.33 & 0.00 \\
Residential (25 mph) & 38.10 & 14.29 \\
Rural (55 mph) & 33.33 & 14.29 \\
Urban (35 mph) & 9.52 & 42.86 \\
\hline
\end{tabular}

TABLE 8 Comparing Individual Average Speeds (percentage of drivers)

\begin{tabular}{lcc}
\hline Segment & AVSAS $<$ Warning & AVSAS $<$ Mandatory \\
\hline Freeways (65 mph) & 57.14 & 42.86 \\
Collector (45 mph) & 47.62 & 38.10 \\
Local (35 mph) & 42.86 & 19.05 \\
Residential (25 mph) & 47.62 & 42.86 \\
Rural (55 mph) & 33.33 & 28.57 \\
Urban (35 mph) & 14.29 & 23.81 \\
\hline
\end{tabular}

were found to be generally higher with AVSAS than with the two ISA systems.

\section{Consumer Acceptance of AVSAS Relative to Other ISA Systems}

The drivers were asked at the end of the experiment to identify whether they would be willing to accept installation of any of the systems in their vehicles as standard equipment. The responses, as shown in Figure 6, demonstrate that a majority of the drivers (76.2\%) would be willing to have the information ISA as standard equipment in their vehicles. Drivers preferred the AVSAS (61.9\%) over the mandatory ISA $(23.8 \%)$ as standard equipment in vehicles.

Although more subjects would be willing to have the information ISA installed as standard equipment, fewer would be willing to pay

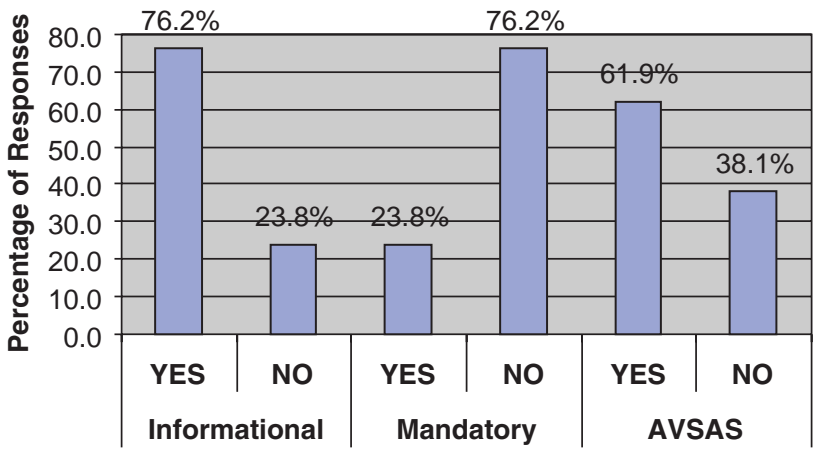

FIGURE 6 Responses to "Would you be willing to have this system installed as standard equipment in your vehicle?"

for an information ISA compared with AVSAS. As shown in Figure 7, the drivers indicated they were more willing to purchase the AVSAS technology (52.4\%) than the other two systems. The participants were the least interested in the purchase of the mandatory ISA (19\%). In addition, the drivers indicated that they most favored having the information-warning ISA (85.7\%) installed if discounts were provided in their auto insurance premiums (see Figure 8 ). The mandatory ISA was the least preferred (42.9\%).

\section{CONCLUSION AND FUTURE WORK}

In this simulator evaluation of AVSAS, the data showed that the technology holds the promise of striking the balance between effectiveness and acceptability. On average, maximum and average speeds were reduced using AVSAS compared with NOISA by $5 \%$ and $3 \%$, respectively. Although the maximum and average speeds were not significantly reduced compared with the mandatory and warning systems, more drivers were willing to purchase the AVSAS technology, even if insurance discounts are not offered.

Future work includes analyzing the effect of the different parameterization of adaptable algorithms underlying AVSAS. These analyses would more fully examine the extent to which AVSAS is successfully adapting to individual driver styles. Moreover, this analysis could explore the effect of different configurations on driver acceptance and driver speed behavior.

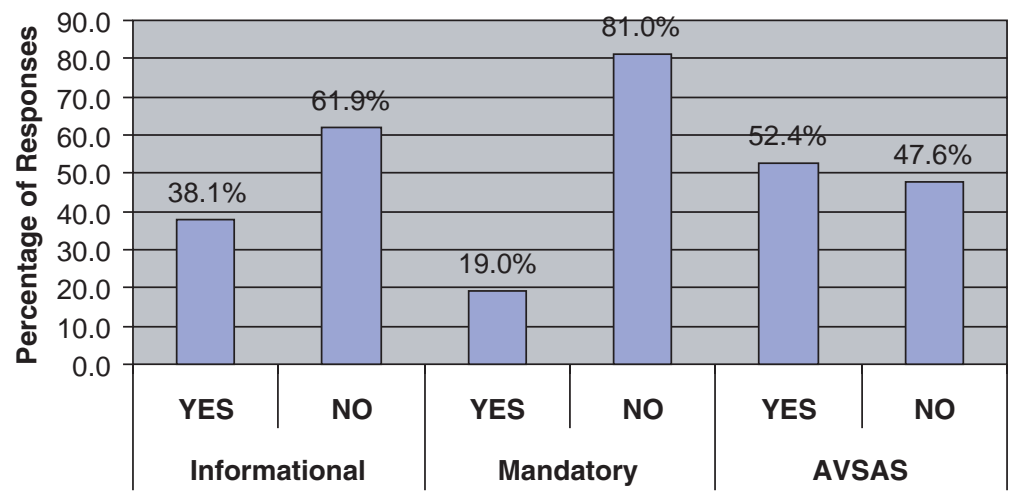

FIGURE 7 Responses to "Would you be willing to purchase this system for your vehicle?" 


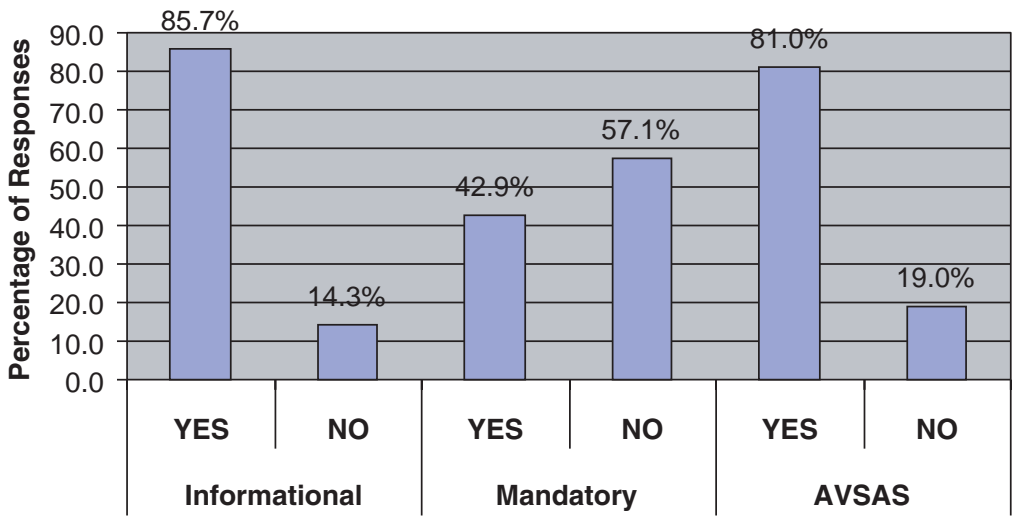

FIGURE 8 Responses to "Would you be willing to use this system if you were to get auto insurance discounts for having this installed in your vehicle?"

This research involved 21 subjects. Although statistical significance was established for some of the reductions in the speed measures, it could be further enhanced by testing the system with additional subjects. Given the different nature of the real highway driver, AVSAS should be analyzed in long-term field operation tests. In addition to providing insight into the effectiveness of the system with the real-world driver, these tests could explore behavioral adaptations, both positive and negative, that might arise from long-term use of AVSAS. In a field operation test, AVSAS could be integrated with vehicle highway systems, as well as with geographical information systems or global positioning system information to enable the actual speed limits on road segments to be used in the underlying architecture.

The effect of any of the ISA systems on crashes and damages could also be addressed and drivers' speeding behaviors for each type of ISA would help with designs of vehicle speed management systems in the future.

\section{ACKNOWLEDGMENT}

Appreciation is extended to FHWA's Turner-Fairbank Highway Research Center, Highway Research Division in McLean, Virginia, for supporting this study.

\section{REFERENCES}

1. National Highway Traffic Safety Administration. Traffic Safety Facts 2003: A Compilation of Motor Vehicle Crash Data from the Fatality Analysis Reporting System and the General Estimates System. National Center for Statistics and Analysis, NHTSA, U.S. Department of Transportation, 2005.

2. Teed, N., A. K. Lund, and R. Knoblauch. The Duration of Speed Reductions Attributable to Radar Detectors. Accident Analysis and Prevention, Vol. 25, No. 2, 1993, pp. 131-137.
3. Comte, S. L., and A. Várhelyi. The Effects of ATT and Non-ATT Systems and Treatments on Driver Speed Behavior. Managing Speeds of Traffic on European Roads. Working Paper R3.1.1 of the MASTER Project, Lund University. Lund, Sweden, 1997.

4. Carsten, O., and F. Tate. Intelligent Speed Adaptation: Accident Savings and Cost-Benefit Analysis. Accident Analysis and Prevention, Vol. 37, 2005, pp. 407-416.

5. Blum, J., and A. Eskandarian. Managing Effectiveness and Acceptability in Intelligent Speed Adaptation Systems. Presented at IEEE Intelligent Transportation Systems Conference, Toronto, Ontario, Canada, 2006.

6. Comte, S. L., and H. Jamson. The Effects of ATT and non-ATT Systems and Treatments on Speed Adaptation Behavior. In Proc., 9th Internal Conference on Road Safety in Europe, Bergisch, Cologne, Germany, 1998.

7. Comte, S. L., and O. M. J. Carsten. Evaluation of In-Car Speed Limiters: Simulator Study. Working Paper R3.2.1 of the MASTER Project, 13th Triennial Congress of the International Ergonomics Association 6, Finnish Institute of Occupational Health, Tampere, Finland, 1998, pp. 302-304.

8. Várhelyi, A., and S. Comte. Evaluation of In-Car Speed Limiters: Managing Speeds of Traffic on European Roads. 5th World Congress on Intelligent Transport Systems, October 1998, Seoul, South Korea. 1998.

9. Päätalo, M., and H. Peltola. Intelligent Speed Adaptation-Effects on Driver Behavior. European Working Group on Speed Control, Aalborg, Denmark, 2001.

10. Biding, T., and G. Lind. Intelligent Speed Adaptation (ISA): Results of Large-Scale Trials in Borlänge, Lidköping, Lund and Umeå During the Period 1999-2002. Ertico, ITS Europe, Brussels, Belgium, 2002.

11. Saad, F., and G. Malaterre. La Regulation de la Vitesse: Analyse des Aides au Controle de la Vitesse. Organisme National de Securité Routière, Arcueil, France, 1982.

12. Carsten, O. M. J., and M. Fowkes. External Vehicle Speed Control: Executive Summary of Project Results. The University of Leeds and Motor Industry Research Association, Leeds, United Kingdom, 2000.

13. Lahrmann, H., and J. R. Madsen. Intelligent Speed AdaptationDevelopment of a GPS Based ISA-System and Field Trial of the System with 24 Test Drivers. Presented at ITS World Congress, Sydney, Australia, 2001.

14. Várhelyi, A., and C. Hydén. The Effects of Large Scale Use of Active Accelerator Pedal in Urban Areas. Presented at International Cooperation on Theories and Concepts in Traffic Safety Workshop on Speed Management Strategies and Implementation, Brno, Czech Republic, 2002.

The Vehicle-Highway Automation Committee sponsored publication of this paper. 